\title{
ON THE SEARCH OF THE "ELUSIVE" INTERMEDIATE MASS BLACK-HOLES
}

\author{
M. D. Caballero-García ${ }^{1}$, S. Fabrika ${ }^{2,3}$, A. J. Castro-Tirado ${ }^{4,5}$, M. Bursa ${ }^{1}$, M. Dovčiak ${ }^{1}$, A. Castellón ${ }^{6}$, \\ and V. Karas ${ }^{1}$
}

\begin{abstract}
Ultra-Luminous X-ray sources (ULXs) are accreting black holes for which their X-ray properties have been seen to be different to the case of stellar-mass black hole binaries. For most of the cases their intrinsic energy spectra are well described by a cold accretion disc (thermal) plus a curved high-energy emission components. The mass of the black hole $(\mathrm{BH})$ derived from the thermal disc component is usually in the range of 100-10 solar masses, which have led to the idea that this can represent strong evidence of the Intermediate Mass Black Holes (IMBH), proposed to exist by theoretical studies but with no firm detection (as a class) so far. Recent theoretical and observational developments are leading towards the idea that these sources are instead compact objects accreting at an unusual super-Eddington regime instead. On the other hand, gravitational waves have been seen to be a useful tool for finding (some of these) IMBHs. We give a brief overview about the recent advent of the discovery of gravitational waves and their relationship with these so far elusive IMBHs.
\end{abstract}

\section{RESUMEN}

Los agujeros negros de masa intermedia (IMBH) son una hipótesis aún no confirmada con total seguridad (como clase de objetos) a partir de evidencias observacionales directas. Su masa sería mayor que la de los agujeros negros de tipo estelar y menor que la de los monstruosos agujeros negros supermasivos. Unos objetos candidatos a rellenar este hueco lo constituyen las fuentes de rayos X ultra-luminosas (ULX) en cuyas emisiones se observan propiedades diferentes al caso de los agujeros negros de masa estelar en sistemas binarios. Sin embargo, desarrollos teóricos y observacionales recientes conducen a la idea de que estas fuentes son, en su lugar, objetos compactos de masa estelar que acretan en un régimen inusual de super-Eddington. Por otro lado, las ondas gravitacionales se han visto como una herramienta útil para encontrar los IMBH. En este artículo damos una breve descripción sobre el descubrimiento de las ondas gravitacionales y su relación con estos agujeros negros de masa intermedia, hasta el momento esquivos.

Key Words: accretion, accretion-discs — black hole physics — cosmology: early universe — galaxies: formation — gravitational waves - X-rays: general

\section{INTRODUCTION}

Ultra-Luminous X-ray sources (ULXs) are pointlike, off-nuclear, extra-galactic sources, with observed X-ray luminosities $\left(\mathrm{L}_{\mathrm{X}} \geq 10^{39} \mathrm{erg} \mathrm{s}^{-1}\right)$ higher than the Eddington luminosity for a stellar-mass black-hole $\left(\mathrm{L}_{\mathrm{X}} \approx 10^{38} \mathrm{erg} \mathrm{s}^{-1}\right)$. The true nature of

\footnotetext{
${ }^{1}$ Astronomical Institute, Academy of Sciences of the Czech Republic, Boční II 1401, CZ-141 00 Prague, Czech Republic (garcia@asu.cas.cz).

${ }^{2}$ Special Astrophysical Observatory, Nizhnij Arkhyz 369167, Russia.

${ }^{3}$ Kazan Federal University, Kremlevskaya 18, Kazan 420008, Russia.

${ }^{4}$ Instituto de Astrofísica de Andalucía (IAA-CSIC), P.O. Box 03004, E-18080, Granada, Spain.

${ }^{5}$ Departamento de Ingeniería de Sistemas y Automática, Escuela de Ingeniería Industrial, Universidad de Málaga (Unidad Asociada al CSIC), Spain.

${ }^{6}$ Facultad de Ciencias, Dpto. de Álgebra, Geometría y Topología, Universidad de Málaga. Campus de Teatinos, s/n Málaga, Spain.
}

these objects is still debated (Feng \& Soria, 2011; Fender \& Belloni, 2012) as there is still no unambiguous estimate for the mass of the compact object hosted in these systems.

Most ULXs are thought to be powered by superEddington accretion onto a stellar mass black hole which can be accomplished (i) by powering strong disc winds (Shakura \& Sunyaev 1973; Lipunova et al. 1999), (ii) by advecting the radiation along with the flow as in radiation pressure dominated disc models like Polish doughnuts (Abramowicz et al. 1978; Jaroszynski et al. 1980) and slim discs (Abramowicz et al. 1988), or (iii) both, advection and outflows (Poutanen et al. 2007; Dotan et al. 2011). Luminosities up to $10^{41} \mathrm{erg} / \mathrm{s}$ can therefore still be explained by super-Eddington mass accretion rates onto stellar mass black holes which can have maximum masses up to $\sim 80 \mathrm{M}_{\odot}$. These higher mass black holes can be explained by direct collapse of metal poor stars 
(Belczynski et al. 2010). The slim disc incorporates the effects of advection. This means that with rising mass accretion rate an increasing fraction of photons gets trapped in the flow, carried inward, and is partly released at smaller radii. A typical slim disc has higher flux at soft photon energies below the spectral peak and above it in comparison to a standard thin disc (Straub et al. 2011, 2014).

Assuming an isotropic emission, in order to avoid the violation of the Eddington limit, ULXs might be powered by accretion onto Intermediate Mass Black Holes (IMBHs) with masses in the range $10^{2}-10^{5} \mathrm{M}_{\odot}$ (Colbert \& Mushotzky 1999; Greene \& Ho 2007; Farrell et al. 2009). Recently, some studies have shown evidence of some ULXs being Black Hole Binaries (BHBs, e.g. M 82 X-2; Kong et al. 2007; Caballero-García et al. 2013b). Later it was shown that M $82 \mathrm{X}-2$ is a binary but accreting onto a neutron star (Bachetti et al. 2014).

In this paper we give an overview of the current models used in the analysis of the X-ray data from ULXs and on the masses derived by using them. Later we justify the use of the slim-disc model for the proper description of these sources. Finally we discuss on the existence of IMBH and their detections in the electromagnetic spectra and with gravitational waves.

\subsection{The X-ray Properties from Ultra-Luminous $X$-ray Sources}

The spectra of ULXs show a power-law spectral shape in the $3-8 \mathrm{keV}$ spectral range, together with a high-energy turn-over at 6-7 keV (Stobbart et al. 2006; Gladstone et al. 2009; Caballero-García et al. 2010), and a soft excess at lower energies (e.g. Kaaret et al. 2006). This soft excess can be modelled by emission coming from the inner accretion disc and is characterized by a low inner disc temperature of $\approx 0.2 \mathrm{keV}$. This is expected if the black holes in these sources are indeed IMBHs (Miller et al. 2004). Other explanations for the soft excess imply a much smaller mass for the $\mathrm{BH}$ in these sources, based on the idea that the accretion in the disc is not intrinsically standard, in contrast to the majority of BHBs (e.g. see Kajava \& Poutanen 2009).

\subsection{The Standard Accretion Disc Theory}

The low inner disc temperatures found for some ULXs were initially interpreted as an evidence for the presence of IMBH (Miller et al. 2004). In the standard disc-black body model (i.e. Multi-Color Disc Blackbody or MCD; Makishima et al. 1986, 2000), which is an approximation of the real standard accretion disc theory, the bolometric luminosity from the accretion disc is calculated as:

$$
L_{\mathrm{bol}}=4 \pi\left(R_{\mathrm{in}} / \zeta\right)^{2} \sigma\left(T_{\mathrm{in}} / \mathrm{f}_{\mathrm{c}}\right)^{4}
$$

Then the mass of the $\mathrm{BH}$ derived from the inner disc temperature from the accretion disc is calculated as:

$$
\frac{\mathrm{M}_{\mathrm{BH}}}{\mathrm{M}_{\odot}}=20.736 \eta\left(\frac{0.41}{\zeta}\right)^{-2}\left(\frac{1.7 \mathrm{kT} \mathrm{T}_{\mathrm{in}}(\mathrm{keV})}{\mathrm{f}_{\mathrm{c}}}\right)^{-4}
$$

Here $\mathrm{f}_{\mathrm{c}} \approx 1.7$ (Shimura \& Takahara 1995) is the ratio of the color temperature to the effective temperature, or "spectral hardening factor", and $\zeta \approx 0.412$ is a correction factor taking into account the fact that $T_{\text {in }}$ occurs at a radius somewhat larger than $R_{\text {in }}$ (Kubota et al. (1998) give $\zeta=0.412$ ) and $\eta$ the Eddington ratio $\left(\mathrm{L}_{\mathrm{bol}}=\eta \mathrm{L}_{\mathrm{Edd}}\right)$. Setting a typical inner disc temperature of inner disc temperature of $\approx 0.2 \mathrm{keV}$ (see references above) equation 2 gives a typical value of $\mathrm{M}_{\mathrm{BH}}=10^{2}-10^{4} \mathrm{M}_{\odot}$ (taking $\eta=0.01-1$, i.e. subEddington accretion).

\subsection{The Anomalous Regime}

However, it has been seen that in a few BHBs (e.g. XTE J1550-564) when they reach a high luminosity level, the L-T relationship departs from what it has been shown above. Then it is called that they have entered the anomalous regime (Kubota et al. 2001, 2004; Gierliński \& Done 2004).

As shown by Kubota et al. (2001), these sources generally follow the $\mathrm{L}_{\text {disc }} \propto \mathrm{T}_{\max }^{4}$ relation. When reaching a luminosity of several orders of magnitude higher there is a small departure from this: with increasing temperature they seem to be slightly underluminous. This is particularly pronounced above $\mathrm{kT}_{\max }=0.9 \mathrm{keV}$ where a break in the $\mathrm{L}-\mathrm{T}$ relation can be seen (Figure 1). The data above the break is consistent with $\mathrm{L}_{\mathrm{disc}} \propto \mathrm{T}_{\max }^{2}$. Kubota et al. (2004) refer to this as the apparently standard regime, and suggest that it is associated with a transition to a slim disc (Abramowicz et al. 1988). However, Gierliński \& Done (2004) noted that this should only occur above $\mathrm{L}_{\mathrm{Edd}}$ (Abramowicz et al. 1988; Shimura \& Takahara 1995), so it seems more likely to represent a subtle change in the colour temperature correction factor.

A shift of the microquasar GRO J1655-40 to the right from the $L-T$ relation for a $7 \mathrm{M}_{\odot} \mathrm{BH}$ (Orosz \& Bailyn 1997) is probably related to a high spin of the $\mathrm{BH}$ there, which results in a higher disc temperature compared to the Schwarzschild BH. 


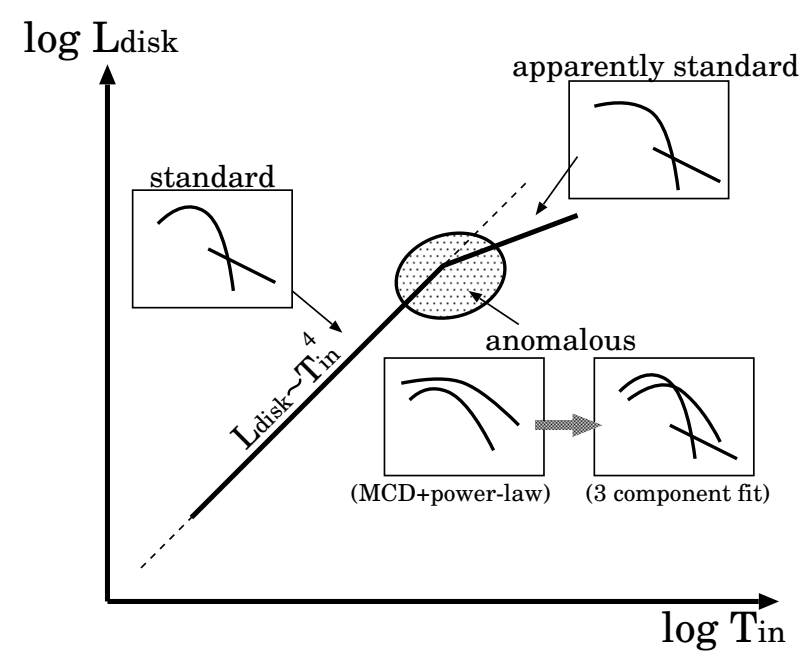

Fig. 1. Schematic picture showing the obtained spectral regimes on the $L_{\mathrm{disc}}-T_{\mathrm{in}}$ diagram. Thick solid and dashed lines show the source behavior obtained under the MCD plus power-law fit.The luminosity-temperature relation for super-critically accreting BHs. From Kubota et al. (2004)

\subsection{The Supercritical Regime}

A recent study of the spectral variability from a sample of ULXs (Kajava \& Poutanen 2009), has shown that the soft excess (i.e. the disc component fitted from the X-ray spectra of some canonical ULXs) does not follow equation 1 but $L_{\mathrm{bol}} \propto T_{\mathrm{in}}^{-3.5}$. This in contrast to what is found for many BHBs (Figure 2) and might indicate that the standard accretion disc theory is not a proper interpretation to the X-ray spectra from ULXs. This implies that the hypothesis on which the IMBH idea is relying (i.e. standard accretion disc theory and the presence of a cold disc) are not valid and it might indicate that these ULXs are not IMBHs as a "class". In the following we develop this idea into more detail.

We have seen above that the standard model for sub-critically accreting BHs (Shakura \& Sunyaev 1973) predicts the relation $L \propto T_{\text {max }}^{4}$. Poutanen et al. (2007) develop a model based on supercritical accretion. It is a model taking into account geometrical effects and where those related to advection are not fully taken into account. In spite of its simplicity it makes the relationship between observable amounts (e.g. temperature and luminosity from the spectra) and those we want to derive (i.e. the mass of the compact object) an easy task. Nevertheless, in the following Sec. we will describe a fully consistent model based on the slim-disc calculations (Abramowicz et al. 1988; Sạdowski et al. 2011; Bursa et al. 2018, in prep.).

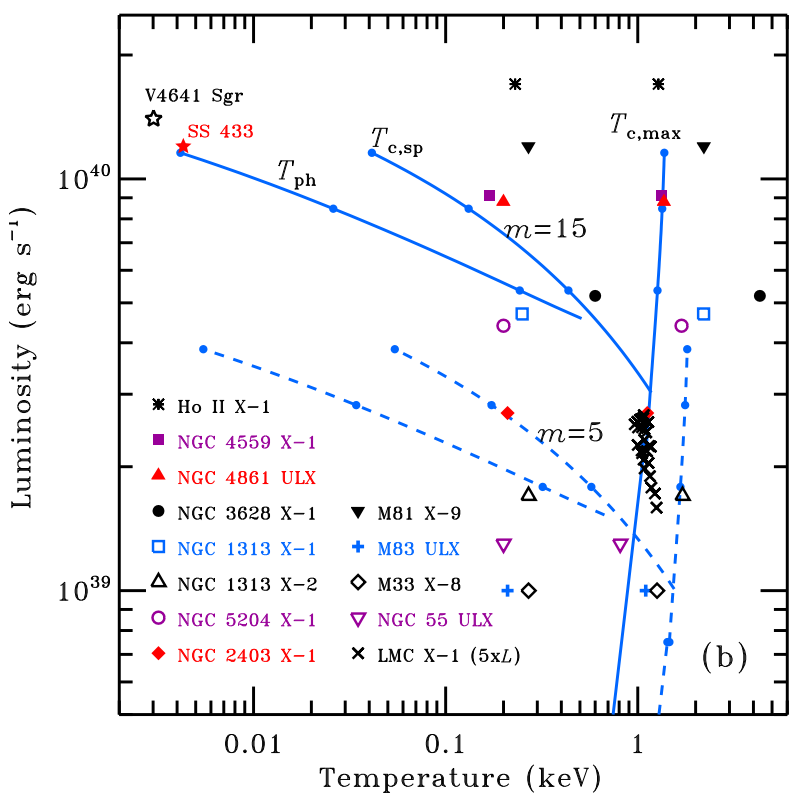

Fig. 2. The luminosity-temperature relation for supercritically accreting BHs. From Poutanen et al. (2007).

At super-Eddington accretion rates, three characteristic temperatures are identified:

-the maximal color disc temperature:

$\mathrm{T}_{\mathrm{c}, \max }=\mathrm{f}_{\mathrm{c}} \mathrm{T}_{\max } \approx 1.6 \mathrm{f}_{\mathrm{c}} \mathrm{m}^{-1 / 4} \mathrm{keV}$,

-the color temperature at the spherization radius:

$\mathrm{T}_{\mathrm{c}, \mathrm{sph}} \approx 1.5 \mathrm{f}_{\mathrm{c}} \mathrm{m}^{-1 / 4} \dot{m}_{0}^{-1 / 2} \mathrm{keV}$,

-the outer photosphere temperature given by:

$\mathrm{T}_{\mathrm{ph}} \approx m^{-1 / 4} \dot{m}_{0}^{-3 / 4} \mathrm{keV}$.

where $\dot{m}_{0}=\dot{\mathrm{M}} / \dot{\mathrm{M}}_{\mathrm{Edd}}$ and $\mathrm{f}_{\mathrm{c}}$ is the correction factor to $\mathrm{T}_{\max }$. They are all marked at Figure 3 .

A soft, $\sim 0.2 \mathrm{keV}$ component may correspond to the spherization temperature implying the accretion rate $\dot{m}_{o}=m^{-1 / 2}\left(1.5 f_{c} / \mathrm{T}_{\mathrm{c}, \mathrm{sph}}[\mathrm{keV}]\right)^{2} \approx 30-40$ onto a stellar mass, $10-20 \mathrm{M}_{\odot}, \mathrm{BH}$. The observed higher luminosities can result from the geometrical beaming. The absolute maximum apparent luminosity using this model (taking all the effects of advection and beaming of the flow) is about $10^{41} \mathrm{ergs}^{-1}$ for a $20 \mathrm{M}_{\odot}$ $\mathrm{BH}$.

\subsection{The Geometry of the Disc}

A face-on observer would see the emission from three separate zones defined by the three characteristic radii (we refer to Poutanen et al. 2007 for more details):

$$
\begin{array}{ll}
r<\mathrm{r}_{\mathrm{ph}, \text { in }}, & \text { zone } \mathrm{A}, \\
\mathrm{r}_{\mathrm{ph}, \text { in }}<r<\mathrm{r}_{\mathrm{sph}}, & \text { zone } \mathrm{B}, \\
\mathrm{r}_{\mathrm{sph}}<r<\mathrm{r}_{\mathrm{ph}, \text { out }}, & \text { zone C. }
\end{array}
$$




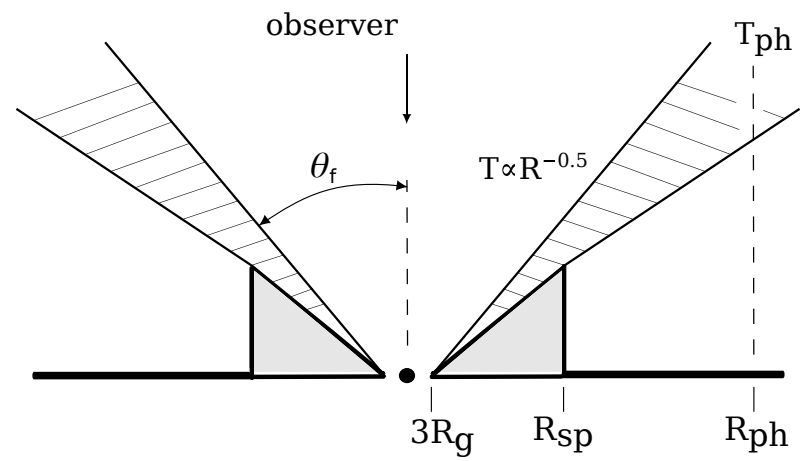

Fig. 3. The model of a supercritical disc with a wind funnel. The figure shows the thin disc $\left(R>R_{\mathrm{sp}}\right)$, the supercritical disc $\left(R \leq R_{\mathrm{sp}}\right)$, and the wind funnel constrained by the radius of the photosphere $R_{\mathrm{ph}} / \sin \theta_{f}$. From Vinokurov et al. (2013).

The characteristic disc temperatures can be obtained from the Stefan-Boltzmann law $\left(\mathrm{Q}_{\mathrm{rad}}(R)=\right.$ $\left.\sigma_{\mathrm{SB}} \mathrm{T}^{4}(R)\right)$.

In zone A (i.e. outside from the photosphere), the wind is transparent (i.e. it is momentum-driven) and the radiation escapes unaffected by the outflow.

In zone $\mathrm{B}$, the wind is opaque and the energy generated in the disc is advected by the wind. The radiation escapes at a radius about twice the energy generation radius. This does not change the radial dependence of the effective temperature $T \propto r^{-1 / 2}$ (i.e. slim-disc configuration),

The outer zone $\mathrm{C}$ emits about the Eddington luminosity which is produced mostly in the disc at radii $r>r_{s p h}$. The photon diffusion time here is smaller than the dynamical time, thus most of the radiation escapes not far from the radius it is produced. This results in the effective temperature variation close to $r^{-3 / 4}$ (i.e. standard accretion regime; Shakura \& Sunyaev 1973).

\subsection{The Need of Slim-Disc Models}

Typical models to fit the X-ray spectra from Ultra-luminous X-ray sources using XSPEC (e.g. DISKBB, DISKPN, KERRBB, BHSPEC, GRAD; Arnaud $1996^{7}$ ) are based on the thin disc model, which is not accurate for $\mathrm{L}>0.3 \mathrm{~L}_{\mathrm{Edd}}$. Indeed, such models tend to give incorrect values for $\mathrm{BH}$ masses and for accretion rates (luminosities) above the Eddington limit, as shown in this section.

The standard (thin) disc follows the $\mathrm{L} \sim \mathrm{T}^{4}$ relation (see equation 1). Nevertheless, advection and obscuration effects cause significant deviations from

\footnotetext{
${ }^{7}$ See the following web page for a list and description of the available models in XSPEC: https://heasarc.gsfc. nasa.gov/xanadu/xspec/manual/.
}

that relation in the super-Eddington regime (Poutanen et al. 2007). These effects are taken into account in the so-called slim-disc models. This effect is strongly inclination dependent (Poutanen et al. 2007) and the luminosity can stay around the Eddington limit even if the mass accretion rate is much higher $\left(\dot{\mathrm{M}} \gg \dot{\mathrm{M}}_{\mathrm{Edd}}\right)$. These facts have implications for the spectral modelling, e.g. getting lower inner disc temperatures given a certain (high) luminosity (Figures 2 and 4). This is in accordance to what has already been observed in the X-ray spectra from sample of representative ULXs (Poutanen et al. 2007).

Instead of using the classical models based on the thin disc theory here we present the results obtained by using the SLIMULX model ${ }^{8}$. SLIMULX (Bursa et al. 2018 , in prep.) is an additive model for thermal continuum emission at high accretion rates to be used with the X-ray spectral-fitting tool XSPEC. The model provides spectral distribution of black-body radiation that is supposed to be emitted from the surface of a slim accretion disc (Abramowicz et al. 1988). A brief description and a first application of the model can be found in Caballero-García et al. (2017).

The major improvement of the SLIMULX model over other commonly used disc models is that it includes three effects that are dominant at high accretion rates, and are not present in the standard Shakura \& Sunyaev (1973); Novikov \& Thorne (1973) disc model:

- Radial advection of heat, which plays a substantial role at higher luminosities, is present and significantly modifies the flux of radiation emitted at a given radius in the inner disc region.

- The inner edge for the disc radiation deviates from ISCO at high luminosity and can be considerably closer to the black hole due to the advective transport of heat generated by viscous processes.

- The location of the effective photosphere differs significantly from the equatorial plane with growing luminosity, although the relative vertical disc thickness is not large $(\mathrm{h} / \mathrm{r} \leq 1)$, not even for the near-Eddington luminosities. Accordingly, the ray-tracing computations are taken from the actual disc photosphere to the observer at infinity (Sa̧dowski et al. 2009).

Bursa et al. (2018, in prep.) fitted the simulated (i.e. faked) SLIMULX spectra with a thin disc model

\footnotetext{
${ }^{8}$ http://stronggravity.eu/results/models-and-data/.
} 
(DISKBB) and the mass was obtained from the fits (Figure 5). At low $\dot{\mathrm{M}}$, the fit recovers the original mass (i.e. the one given by the slim-disc model), but at high mass accretion rate $\left(\dot{\mathrm{M}} \geq 3 \dot{\mathrm{M}}_{\mathrm{Edd}}\right)$ the mass given by the thin disc model is much higher (note that a mass of $10 \mathrm{M}_{\odot}$ was assumed in the SLIMULX simulated spectra). This exercise is representative of what is usually done when fitting the X-ray spectra from ULXs in the literature. It appears quite a bad approach to estimate the $\mathrm{BH}$ parameters when using thin disc models if the disc is strongly radiation pressure dominated.

So we should not use of equations 1 and 2 in the radiation pressure dominated (i.e. in the high accretion rate) regime.

In the SLIMULX model the luminosity consists of the integration of all the radial flux elements (in the so-called corotating frame) multiplied by a factor that accounts for the proper transformation of the corotating frame to the coordinate frame so that the given value is indeed the total luminosity of the disc ignoring geometrical GR effects. In this case the relationship between the mass and any measurable property of the disc is troublesome. Even although the mass is a free parameter of the model, its direct relationship with the rest of quantities is complicated. It is generally assumed that the black hole mass is of a certain value (e.g. $10 \mathrm{M}_{\odot}$ ) when doing explicit calculations. Although relevant disc properties follow a mass scaling law or do not scale with mass, the results can not be directly rescaled and generalized to BH systems of an arbitrary mass, because the output of radiative transfer calculation would be different and the predicted spectra also. The application to stellar mass BH systems is fine over an usually assumed mass range for such systems (few to few tens of solar masses). Note that higher masses (e.g. IMBHs) can be used (and derived) with this model as well (see Straub et al. 2014).

\subsection{On the Masses Derived for the Compact Object Using Slim-Disc Models}

It has been suggested that ULXs appear very luminous due to a combination of moderately high mass (IMBHs), mild beaming and mild superEddington emission and that ULXs are an inhomogeneous population composed of more than one class (Colbert \& Mushotzky 1999; Fabbiano et al. 2006).

Initially, they were supposed to be the IMBHs originating from low-metallicity Population III stars (Madau \& Rees 2001). Nevertheless, they are not spatially distributed throughout galaxies as it would be expected. On the other hand, IMBHs may be
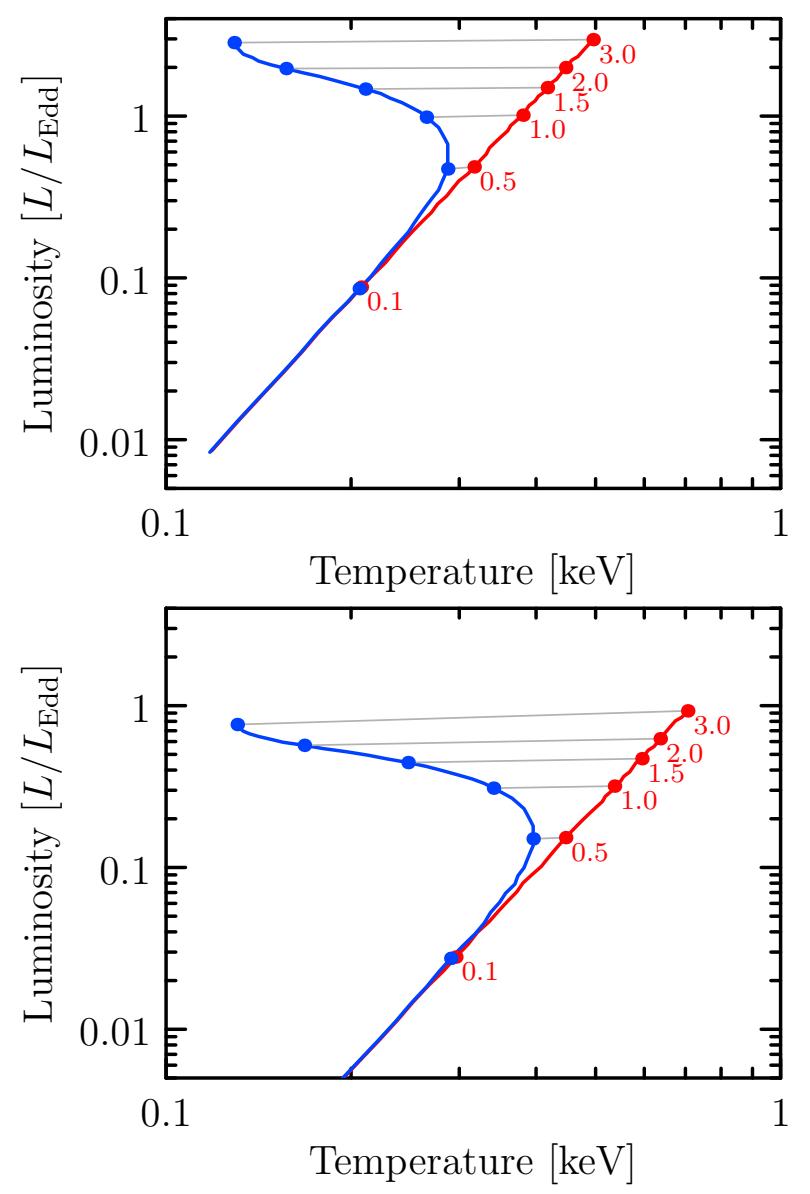

Fig. 4. The luminosity-temperature relation for supercritically accreting BHs. The two panels correspond to two different inclination angles $\left(i=0^{\circ}, 70^{\circ}\right.$, at top and bottom, respectively) of the line of sight to the observer. Blue/red branches correspond to standard Novikov-Thorne (red) and slim (blue) accretion disc, with the labels indicating the accretion rate (in units of Eddington accretion rate). On the horizontal axis, the temperature $[\mathrm{keV}]$ denotes the best-fit temperature from both models (this temperature corresponds to the one giving the maximum emission flux in the calculated spectra). From Bursa et al. (2018, in prep.).

produced in runaway mergers in the cores of young clusters (Portegies Zwart et al. 2004). In such cases, they usually should stay within their clusters. Nevertheless, it has been found (Poutanen et al. 2013) that all brightest X-ray sources in the Antennae galaxies are located nearby the very young stellar clusters. NGC $5408 \mathrm{X}-1$ is also located nearby a young stellar association (Grisé et al. 2012). These studies concluded that these sources were ejected in the process of formation of stellar clusters in the dynamical fewbody encounters and that the majority of ULXs are massive X-ray binaries with the progenitor masses 


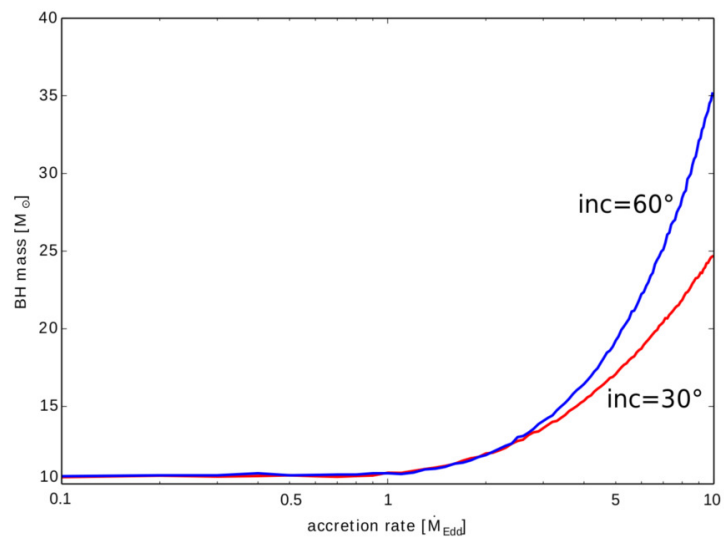

Fig. 5. Simulated SLIMULX spectra are fitted with a thin disc model (DISKBB; Mitsuda et al. 2004; Makishima et al. 1986) and the mass is obtained from the fit (in units of $\mathrm{M}_{\odot}$ ). The horizontal axis is the accretion rate (in units of $\dot{M}_{\mathrm{Edd}}$ ). From Bursa et al. (2018, in prep.).

larger than $50 \mathrm{M}_{\odot}$. Currently, it is thought that only a handful of ULXs could be considered as potential IMBHs (ESO 243-49 HLX-1 between a few others; Farrell et al. 2009; Sutton et al. 2012; Mezcua et al. 2017).

Currently the most accepted idea is that the majority of ULXs are powered by accretion onto stellarmass black holes $\left(<100 \mathrm{M}_{\odot}\right)$ at around or in excess of the Eddington limit (e.g. Colbert \& Mushotzky 1999; Fabrika \& Mescheryakov 2001; King et al. 2001; Fabbiano et al. 2006; Poutanen et al. 2007; Liu et al. 2013).

Due their brightness, most of them are believed to be BHs. However, recently a new class of ULXs was discovered, through the detection of coherent pulsations: Ultra-luminous X-ray pulsars (ULPs). The presence of pulsations unambiguously identifies the compact objects as neutron stars, which are typically less massive than black holes. In ULPs the neutron star accretes matter from a companion star at inferred rates much higher than previously expected. Currently three of these systems are known: M82 X-2 (Bachetti et al. 2014), NGC 5907 ULX (Israel et al. 2017a), and NGC 7793 P13 (Israel et al. 2017b). Furthermore, a clear path forward to obtain a full sample of the ULP population is missing.

In this paper we have described all the models used to describe the data from ULXs, from assuming low accretion rates (and then high $\mathrm{BH}$ mass) to highly super-Eddington accretion rates (then low compact object mass). Applying the best models based on slim-disc configuration and including advection in the high accretion rate favour either low masses for the compact object $\left(\mathrm{M}<30 \mathrm{M}_{\odot}\right.$, e.g. LMC X-3 and NGC5408 X-1 Straub et al. 2011; Caballero-García et al. 2017) or high masses $\left(\mathrm{M} \gtrsim 10^{3} \mathrm{M}_{\odot}\right.$, ESO 243-49 HLX-1 Straub et al. 2014). Therefore $\mathrm{BH}$ masses in the range of $\mathrm{M}=100-$ $10^{3} \mathrm{M}_{\odot}$ are hardly to find in the ULX population. Even though initially this population of ULXs was initially thought to contain a significant part of these IMBHs.

Indeed, to our knowledge, there is no unambiguous detection of the electromagnetic counterpart of a $B H$ with a mass in the range of $\mathrm{M}=80-10^{3} \mathrm{M}_{\odot}$.

\subsection{X-ray Timing Properties of ULXs}

Black Hole masses scale with the break frequency of their Power Density Spectrum (PDS; McHardy et al. 2006; Körding et al. 2007). This relation holds over six orders of magnitude in mass, i.e. from Black Hole Binaries (BHBs) to Super-Massive Black Holes (SMBHs).

The PDS and the energy spectra of NGC 5408 $\mathrm{X}-1$ (Figure 6 ) and $\mathrm{M} 82 \mathrm{X}-1$ (for instance) are very similar to that of BHBs in the Steep Power-law state (i.e. one of the so-called intermediate states). Nevertheless, the characteristic time-scales within the PDS are lower by a factor of $\mathrm{a} \approx 100$ and the X-ray luminosity is higher by a factor of a few $\approx 10$, when compared to BHBs. This gives $\mathrm{BH}$ mass estimates for these ULXs of the order of $\mathrm{M}_{\mathrm{BH}} \gtrsim 10^{3}-10^{4} \mathrm{M}_{\odot}$, from their X-ray timing properties only, as explained in the following.

As described in the previous Sections, determining the mass from the $\mathrm{BH}$ in ULXs has been the goal of several studies. For example, in NGC 5408 $\mathrm{X}-1$ there is still no consensus on whether it is an IMBH or a stellar-mass BH. Previous estimates from the timing properties (Strohmayer et al. 2009; Dheeraj \& Strohmayer 2012) indicate a mass of $\mathrm{M}_{\mathrm{BH}} \gtrsim 1000 \mathrm{M}_{\odot}$, thus an IMBH, but others (Middleton et al. 2011) indicate a much smaller mass of $\mathrm{M}_{\mathrm{BH}} \leq 100 \mathrm{M}_{\odot}$, thus a stellar-mass $\mathrm{BH}^{9}$. In the first case considered the accretion rate is sub-Eddington, whilst the latter case indicates (near or) superEddington accretion.

If the Quasi-Periodic Oscillation (QPO) detected in $\mathrm{M} 82 \mathrm{X}-1$ is a fundamental High Frequency QPO (HFQPO), then it appears at much lower frequency $(\approx 50 \mathrm{mHz})$ than those observed in BHBs $(35-450 \mathrm{~Hz})$, by three orders of magnitude. Scaling linearly with the mass of a stellar-mass $\mathrm{BH}\left(10 \mathrm{M}_{\odot}\right)$

\footnotetext{
${ }^{9}$ It has to be noted here that in low metallicity environments BHs with masses up to $80-130 \mathrm{M}_{\odot}$ can still be formed through direct stellar-collapse (Zampieri et al. 2009; Belczynski et al. 2010) and this is why we are referring to them as stellar-mass BHs.
} 


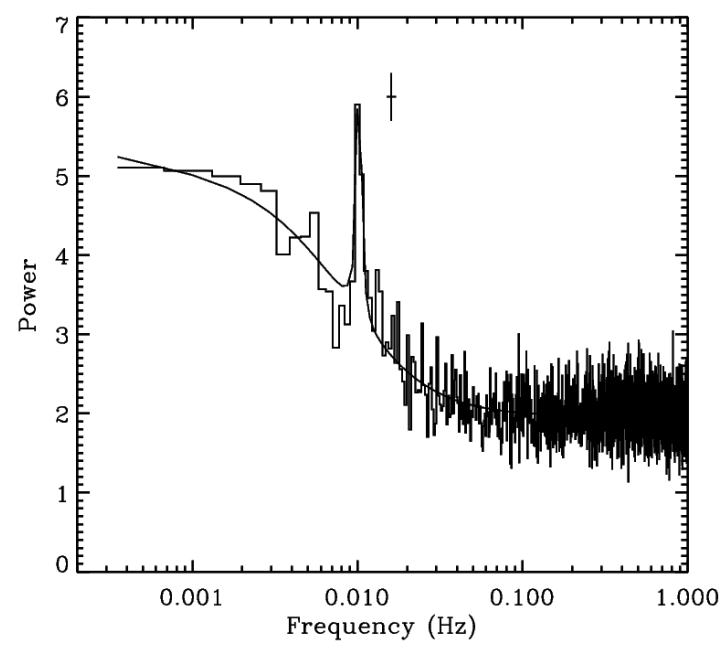

Fig. 6. Average Power Density Spectrum of NGC 5408 X-1. From Strohmayer et al. 2009.

if these QPOs have the same origin, the frequencies we have found lead to a mass of $\mathrm{M}_{\mathrm{BH}} \approx\left(10^{4}-10^{5}\right) \mathrm{M}_{\odot}$ (taking into account the whole range of possible values of the spin of the $\mathrm{BH}$ ) for the mass of the $\mathrm{BH}$ in $\mathrm{M} 82 \mathrm{X}-1$. Other mass estimates, this time based on spectral-timing scaling relationships from systems of different mass (Titarchuk \& Fiorito 2004), have provided a mass estimate of $\approx 10^{3} \mathrm{M}_{\odot}$ (Fiorito \& Titarchuk 2004). Nevertheless, caution is required in order to apply relationships based solely on the mass of the BH systems. As already pointed out by several authors (McHardy et al. 2006 for AGNs; Soria 2007 and Casella et al. 2008 for ULXs) the accretion rate is an important parameter that, together with the mass of the $\mathrm{BH}$, should be considered as the main drivers in these scaling relationships. This effect could lead to a much smaller mass for the BH (compact object). In addition, it remains to date unclear how timing properties and $\mathrm{BH}$ masses are related, and what are the properties of the accretion states in ULXs compared to those of Galactic BHBs (Caballero-García et al. 2013a; Atapin et al. 2018, in prep.).

\section{GRAVITATIONAL WAVES AS A NEW WINDOW TO THE UNIVERSE}

As reported by Abbott et al. (2016) the first gravitational-wave $(\mathrm{GW})$ transient was identified in data recorded by the Advanced Laser Interferometer Gravitational-wave Observatory (LIGO) detectors on 2015 September 14. The event, initially designated G184098 and later given the name GW150914, is described in detail elsewhere. By prior arrangement, preliminary estimates of the time, significance, and sky location of the event were shared with 63 teams of observers covering radio, optical, nearinfrared, X-ray, and gamma-ray wavelengths with ground- and space-based facilities. As this event turned out to be a binary black hole merger, there is little expectation of a detectable electromagnetic (EM) signature. GW150914 is consistent with the inspiral and merger of two BHs of masses $36_{-4}^{+5}$ and $29 \pm 4 \mathrm{M}_{\odot}$, respectively, resulting in the formation of a final $\mathrm{BH}$ of mass $62 \pm 4 \mathrm{M}_{\odot}$ at a distance of $410_{-180}^{+160} \mathrm{Mpc}$.

As reported by Abbott et al. (2017) on 2017 August 17 a binary neutron star coalescence candidate (later designated GW170817) with merger time 12:41:04 UTC was observed through gravitational waves by the Advanced LIGO and Advanced Virgo detectors. The Fermi Gamma-ray Burst Monitor independently detected a gamma-ray burst (GRB $170817 \mathrm{~A}$ ) with a time delay of $\approx 1.7 \mathrm{~s}$ with respect to the merger time. From the gravitational-wave signal, the source was initially localized to a sky region of $31 \mathrm{deg}^{2}$ at a distance of $40_{-8}^{+8} \mathrm{Mpc}$ and with component masses consistent with neutron stars. The component masses were later measured to be in the range 0.86 to $2.26 \mathrm{M}_{\odot}$. An extensive observing campaign was launched across the electromagnetic spectrum leading to the discovery of a bright optical transient (SSS17a, now with the IAU identification of AT 2017gfo) in NGC 4993 less than 11 hours after the merger. The follow-up observations support the hypothesis that GW170817 was produced by the merger of two neutron stars in NGC 4993 followed by a short gamma-ray burst (GRB 170817A) and a kilonova/macronova powered by the radioactive decay of r-process nuclei synthesized in the ejecta.

In Figure 7 there is an interactive graphic featuring all the $\mathrm{BH}$ detected by LIGO (including GW170608), and the recently announced Neutron Stars, plus all the other compact objects known from electromagnetic measurements.

\subsection{Detection of the Missing IMBHs}

Black holes, the ultra-compact remnants of very massive stars, are prime candidates for emitting detectable gravitational waves in this way. While current detection work focuses on commonly-expected binary systems of black holes with masses a few times that of the Sun, it is possible that there are detectable populations of black holes which have masses larger than this, i.e. hundreds or thousands times the mass of the Sun. These are dubbed IMBHs, as their masses lie in the currentlyunobserved range between solar mass black holes and the supermassive black holes in the center of galaxies. The gravitational-wave interferometer network 


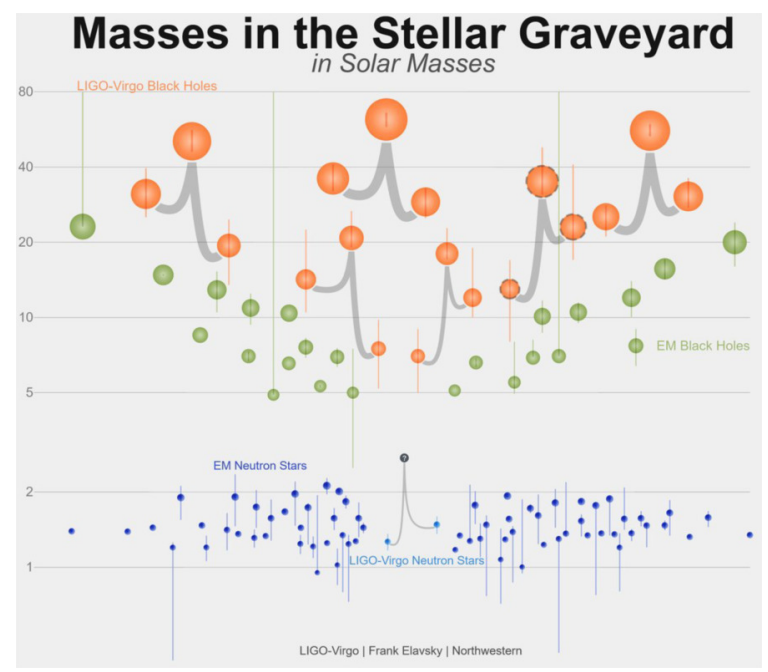

Fig. 7. Interactive graphic featuring all of the Black Holes (including GW170608), the recently announced Neutron Stars, and all the other compact objects known from electromagnetic measurements. From LIGO collaboration.

of LIGO and Virgo are able to measure ripples from the final moments of such intermediate mass binary black holes merging together.

Candidate IMBHs, nevertheless are still relatively rare (see our discussion above). Gravitationalwave detection of intermediate mass black hole binary mergers confirm their existence and provide information on the abundance of such systems in the Universe, as well as precisely measuring the component masses. The presence or absence of these signals will shed light on star formation in the early universe, provide important information about the dynamics and structures of globular clusters, and give clues about the formation of supermassive black holes.

Black holes are just that - black. They are the corpses of dead stars, so massive and compact that not even light can escape them. They literally cannot be seen, and before LIGO came online, their existence could only be inferred by their gravitational effect on their neighbors or because of light (often Xrays) emitted by hot gas falling into the black hole.

But an isolated black hole is invisible. It interacts via gravity and, even then, it only emits gravitational radiation when it is moving. So detectors like LIGO or Virgo are the only way to see them. They are essentially black hole telescopes.

With even just a few observations of gravitational waves, the LIGO measurements are perplexing. Prior to 2016, it was thought that there were two classes of black holes: stellar-mass black holes, with masses no more than about 10 times that of our
Sun, and massive, monstrous black holes at the center of galaxies with masses in the range of hundreds of thousands to billions of solar masses.

Black holes with masses in the range of $\gtrsim 30-$ $10^{3} \mathrm{M}_{\odot}$ were unexpected. And yet, that's just what LIGO (and now LIGO plus Virgo) have observed. It has been shown that $\mathrm{BHs}$ in the mass range of $30-130 \mathrm{M}_{\odot}$ can be formed through $\mathrm{BH}-\mathrm{BH}$ mergers in low-metallicity environments (see recent simulations from Marchant et al. 2016, 2017). They could justify the discovery of the recent IMBHs discovered by LIGO so far $\left(\leq 130 \mathrm{M}_{\odot}\right.$; Figure 7$)$. But observationally, IMBHs with masses in the range $100-10^{3} \mathrm{M}_{\odot}$ are extremely rare to find. They could form (part of) the unseen dark matter proposed to exist in the galactic haloes by cosmological studies (see e.g. Mediavilla et al. 2017, who tested for this possibility).

\section{DISCUSSION AND CONCLUSIONS}

Ultra-Luminous X-ray sources (ULXs) are accreting black holes for which their X-ray properties have been seen to be different to the case of stellarmass black hole binaries. For most of the cases their intrinsic energy spectra are well described by a cold accretion disc (thermal) plus a curved high-energy emission components. The mass of the black hole $(\mathrm{BH})$ derived from the thermal disc component is usually in the range of $100-10^{5} \mathrm{M}_{\odot}$, which have led to the idea that this might represent strong evidence of the Intermediate Mass Black Holes (IMBH), proposed to exist by theoretical studies but with no firm detection (as a class) so far. But the mass estimation depends on the accretion rate.

We have discussed the masses obtained by using different models considering different accretion rate regimes. Masses of the IMBH in the range of $\mathrm{M}=80-10^{3} \mathrm{M}_{\odot}$ are hardly to obtain. Also, recent theoretical and observational developments are leading towards the idea that ULXs are instead stellarmass compact objects accreting at an unusual superEddington regime instead, therefore favouring lower mass estimates for the compact objects.

On the other hand, gravitational waves have been seen to be a useful tool for finding (some of) these IMBHs. We have given a brief overview about the recent advent of the discovery of gravitational waves and their relationship with these so far elusive IMBHs.

Acknowledgments: MCG, MB and MD acknowledge support provided by the European Seventh Frame-work Programme (FP7/2007-2013) under grant agreement $n^{\circ} 312789$ and GA CR grant 
18-00533S. SF acknowledges support by the Russian Science Foundation (N 14-50-00043) and the Russian Government Program of Competitive Growth of KFU. AJCT acknowledges support from the Spanish MINECO Ministry project: AYA2015-71718-R.

\section{REFERENCES}

Abbott, B. P., Abbott, R., Abbott, T. D., et al. 2016, ApJ, 826, 13

Abbott, B. P., Abbott, R., Abbott, T. D., et al. 2017, ApJ, 848, 12

Abramowicz, M., Jaroszynski, M., \& Sikora, M. 1978, A\&A, 63, 221

Abramowicz, M. A., Czerny, B., Lasota, J. P., \& Szuszkiewicz, E. 1988, ApJ, 332, 646

Arnaud, K. A., 1996, ASPC, 101, 17

Atapin, K., Fabrika, S. \& Caballero-Garcia, M. D., 2018, in prep.

Bachetti, M., et al. 2014, Natur, 514, 202

Belczynski, K., Bulik, T., Fryer, C. L., et al. 2010, ApJ, 714,1217

Bursa, M., et al. 2018, in prep.

Caballero-García, M. D. \& Fabian, A. C., 2010, MNRAS, 402, 2559

Caballero-García, M. D., Belloni, T. M., \& Wolter, A., 2013a, MNRAS, 435, 2665

Caballero-García, M. D., Belloni, T., \& Zampieri, L. 2013b, MNRAS, 436, 3262

Caballero-Garcia, M. D., Bursa, M., Dovčiak, M. et al. 2017, CoSka, 47, 84

Casella, P., Ponti, G., Patruno, A., et al. 2008, MNRAS, 387, 1707

Colbert, E. J. M. \& Mushotzky, R. F. 1999, ApJ, 519, 89

Dheeraj, P. R. \& Strohmayer, T. E. 2012, ApJ, 753, 139

Dotan, C. \& Shaviv, N. J. 2011, MNRAS, 413, 1623

Ebisawa, K., Mitsuda, K., \& Hanawa, T. 1991, ApJ, 367, 213

Fabbiano, G. 2006, ARA\&A, 44, 323

Fabrika, S. \& Mescheryakov, A. 2001, IAUS, 205, 268

Farrell, S. A., Webb, N. A., Barret, D., et al. 2009, Natur, 460,73

Fiorito, R. \& Titarchuk, L. 2004, ApJ, 614, 113

Gierliński, M. \& Done, C. 2004, MNRAS, 347, 885

Gladstone, J. C., Roberts, T. P., \& Done, C. 2009, MNRAS, 397, 1836

Greene, J. E. \& Ho, L. C. 2007, ApJ, 670, 92

Grisé, F., Kaaret, P., Corbel, S., et al. 2012, ApJ, 745, 123

Hutchings, J. B., Crampton, D., \& Cowley, A. P. 1983, ApJ, 275, 43

Israel, G. L., Belfiore, A., Stella, L., et al. 2017, Sci, 355, 817

Israel, G. L., Papitto, A., Esposito, P., et al. 2017, MNRAS, 466, 48

Jaroszynski, M., Abramowicz, M. A., \& Paczynski, B. 1980, AcA, 30, 1

Kaaret, P., Simet, M. G., \& Lang, C. C. 2006, ApJ, 646, 174
Kajava, J. J. E. \& Poutanen, J. 2009, MNRAS, 398, 1450

King, A. R., et al. 2001, ApJ, 552L, 109

Kong, A. K. H., et al. 2007, ApJ, 671, 349

Körding, E. G., Migliari, S., Fender, R., et al. 2007, MNRAS, 380, 301

Kubota, A., et al. 1998, PASJ, 50, 667

Kubota A., Makishima K., \& Ebisawa K. 2001, ApJ, 560, L147

Kubota, A. \& Makishima, K. 2004, ApJ, 601, 428

Lipunova, G. V. 1999, AstL, 25, 508

Liu, J.-F., et al. 2013, Natur, 503, 500

Madau, P. \& Rees, M. J. 2001, ApJ, 551, 27

Makishima, K., et al. 1986, ApJ, 308, 635

Makishima, K., et al. 2000, ApJ, 535, 632

Marchant, P., Langer, N., Podsiadlowski, P., et al. 2016, A\&A, 588, 50

Marchant, P., Langer, N., Podsiadlowski, P., et al. 2017, A\&A, 604, 55

McHardy, I. M., Koerding, E., \& Knigge, C. 2006, Natur, 444, 730

Mediavilla, E., Jiménez-Vicente, J., Muñoz, J. A., et al. 2017, ApJ, 836, 18

Mezcua, M. 2017, IJMPD, 26, 30021

Middleton, M. J., Roberts, T. P., Done, C., et al. 2011, MNRAS, 411, 644

Miller, J. M., Fabian, A. C., \& Miller, M. C. 2004, ApJ, 607, 931

Mitsuda, K., Inoue, H., Koyama, K., et al. 1984, PASJ, 36,741

Novikov, I. D. \& Thorne, K. S. 1973, Astrophysics of black holes, edited by C. DeWitt \& B. DeWitt (New York, NY: Gordon and Breach)

Orosz, J. A. \& Bailyn, C. D. 1997, ApJ, 477, 876

Portegies Zwart, S. F., Dewi, J., \& Maccarone, T. 2004, MNRAS, 355, 413

Poutanen, J., Lipunova, G., Fabrika, S., Butkevich, A. G., \& Abolmasov, P. 2007, MNRAS, 377, 1187

Poutanen, J., Fabrika, S., Valeev, A. F., et al. 2013, MNRAS, 432, 506

Sa̧dowski, A. 2009, ApJS, 183, 171

Sądowski, A., et al. 2011, A\&A, 527A, 17

Shakura, N. I. \& Sunyaev, R. A. 1973, A\&A, 24, 337

Shimura, T. \& Takahara, F. 1995, ApJ, 445, 780

Soria, R. 2007, Ap\&SS, 311, 213

Stobbart, A.-M., Roberts, T. P. \& Wilms, J. 2006, MNRAS, 368, 397

Straub, O., Bursa, M., Sądowski, A., et al. 2011, A\&A, 533,67

Straub, O., Godet, O., Webb, N., et al. 2014, A\&A, 569, 116

Strohmayer, T. E. \& Mushotzky, R. F. 2009, ApJ, 703, 1386

Sutton, A. D., Roberts, T. P., Walton, D. J., et al. 2012, MNRAS, 423, 1154

Titarchuk, L. \& Fiorito, R. 2004, ApJ, 612, 988

Vinokurov, A., Fabrika, S., \& Atapin, K. 2013, AstBu, 68,139

Zampieri, L. \& Roberts, T. P. 2009, MNRAS, 400, 677 